\title{
Neighboring Optimal Control for Mobile Robot Trajectory Tracking with Range-Limited Sensors
}

\author{
Suruz Miah, Wail Gueaieb, and Davide Spinello \\ School of Electrical Engineering and Computer Science \\ University of Ottawa \\ Ottawa, Ontario, K1N6N5, Canada \\ e-Mails: \{Suruz.Miah,Wail.Gueaieb,Davide.Spinello\}@uOttawa.ca
}

\author{
Kaisar Khan \\ Department of Electrical Engineering \\ McNeese State University \\ 4205 Ryan Street, Lake Charles, LA \\ e-Mail: mkhan@mcneese.edu
}

\begin{abstract}
Among the major challenges in tracking a predefined trajectory of a nonholonomic mobile robot operating in indoor environments is to determine an appropriate feedback control. In the technical literature, numerous controllers have been proposed to date for solving trajectory tracking and/or regulation problems of mobile robots. Most of them are quite promising to implement and mobile robots can operate in quite complex environments with these controllers. Nevertheless, one of the shortcomings of most of the existing controllers is the requirement of sophisticated hardwares, which, in some cases, more costly than the mobile robot itself. In addition, the analytical expression for most of the controllers is quite complex even for a simple nonholonomic system, an unicycle, for example. In this paper, we propose a neghboring optimal controller coupled with the state estimation for a differential drive mobile robot to track a pre-defined trajectory using signal strength measurements from RF (radio frequency) sensors placed in its operating environment. We emphasize that the RF sensors have limited communication range with the robot. The proposed controller is easy to implement, does not require sophisticated hardware for processing, and has relatively easier expression for the feedback controller. A numerical example is provided to validate the theoretical results. Computer simulations will be backed by experimental results for future investigations.
\end{abstract}

\section{INTRODUCTION}

Tracking problems have been addressed in a variety of robotic platforms [1], [2], [3], [4], [5] using intelligent control laws coupled with adaptation techniques. Among them, regulating nonholonomic wheeled mobile robots to track a pre-defined trajectory or to reach a fixed configuration in an operating environment is a challenging task. This task is often performed in cooperation with the robot's state estimation for a real-time implementation. In most cases, it is assumed that the robot is always able to receive measurements from all sensors at all times, see [6] and some references therein. In [6], we addressed the trajectory tracking problem of a differential drive mobile robot by developing an offline nominal statefeedback controller followed by an online neighboring optimal controller. The latter controller requires that the robot is able to estimate its position and orientation using sensory measurements (signal strengths) from RF nodes placed in the operating environment. Realistically, the robot can receive signal strength measurements from a subset of sensor nodes due to its range-limited communication capability or data packet loss. A practical alternative solution for the robot to track a predefined trajectory under such situations is well motivated. Here we propose a neighboring optimal controller coupled with the robot's state estimation using signal strength measurements from range limited RF sensors placed in its operating environment.

In the technical literature, the trajectory tracking problem of mobile robots has been solved using nonlinear control laws, see [7], [8], [9] for backstepping methods, [10], [11], [12] for sliding mode control, [13], [14], [15] for moving horizon $\mathcal{H}_{\infty}$ tracking control coupled with disturbance effect, and [16] for transverse function approach. A vector-field orientation feedback control method for a differentially driven wheeled vehicle has been demonstrated in [17]. RFID (Radio Frequency Identification) technology drew the attention of a large body of research on mobile robot localization owing to its wide availability, contactless recognition ability, and affordability [18], [19]. In most cases, RFID systems are deployed for solving localization problem (not stabilization or tracking problems) of mobile robots in a particular environment [20], [21], [22], [23]. A sliding mode controller in cooperation with RFID system is proposed in [24] to track a desired trajectory, where RFID tags are placed on the floor in a triangular pattern to estimate the position of the mobile robot. This technique, however, is not suitable if the operating environment is dynamically changed. In 2008, Gueaieb and Miah pioneered a navigation algorithm, where the phase difference of RFID signals is exploited to navigate a mobile robot in an indoor environment [25]. The navigation system is, however, based on a customized RFID reader (not RFID tag) architecture and the navigation performance is evaluated using computer simulations. Moreover, the robot's trajectory tracking and stabilization problems were not explicitly solved in our previous work.

By extending the work in [6], we implement a neighboring optimal controller coupled with state estimation for a differentially drive mobile robot receiving signal strength measurements from a subset of RF sensors placed in its operating environment. The robot's actuator noise and signal strength measurement noise are taken into consideration while implementing this controller. Signal strength measurements emitted from RF sensor are used to better estimate the robot's pose by incorporating an optimal filter. The work 
described herein is tested using a differentially drive mobile robot (DDMR) operating in an indoor environment where RF sensors are placed at $2-\mathrm{D}$ positions.

The rest of the paper is outlined as follows. Section II illustrates the mathematical modeling of a differential drive mobile robot and its range-limited sensory capabilities. Section III formulates the measurement feedback law for the rangelimited sensors. The proposed neighboring optimal control law coupled with the robot's state estimation based on rangelimited sensors is detailed in section IV. Section V gives the thorough evaluation of the current work with some numerical simulation results. Finally, conclusions are drawn in section VI.

\section{Mobile Robot And RF Sensor Modeling}

Following [6], let $(x, y)$ and $\theta$ be the position and the heading angle of a robot with respect to a ground-fixed inertial reference frame $X-Y$. The rotational velocities of the robot's left and right wheels are characterized by the sole (scalar) axis angular velocities $u_{L}$ and $u_{R}$, respectively. The robot's position is the midpoint of the wheelbase of length $l$ connecting the two lateral wheels along their axis.

Let $t_{f}$ be the final time to complete the robot's mission and $\mathcal{I} \equiv\left[0, t_{f}\right]$ denotes the time interval. At time $t \in \mathcal{I}$, the robot kinematic model is given by

$$
\dot{\mathbf{q}}(t)=\mathbf{f}[\mathbf{q}(t), \mathbf{u}(t)]=\frac{r}{2} \mathbf{B}[\mathbf{q}(t)] \mathbf{u}(t),
$$

where $r$ is the robot's wheel radius, $\mathbb{R}^{2} \times \mathbb{S}^{1} \supset \mathcal{Q} \ni$ $\mathbf{q}(t) \equiv[x(t), y(t), \theta(t)]^{T}$ is the robot's configuration in the configuration space, $\mathbb{R}^{2} \supset \mathcal{U} \ni \mathbf{u}(t) \equiv\left[u_{R}(t), u_{L}(t)\right]^{T}$ is its control input, $\theta(t) \in[-\pi, \pi)$,

$$
\mathbf{B}[\mathbf{q}(t)]=\left[\begin{array}{cc}
\cos \theta(t) & \cos \theta(t) \\
\sin \theta(t) & \sin \theta(t) \\
\frac{2}{l} & -\frac{2}{l}
\end{array}\right] .
$$

Since the robot itself is subjected to the noisy speed, the model (1) can be rewritten as

$$
\dot{\mathbf{q}}(t)=\mathbf{f}[\mathbf{q}(t), \mathbf{u}(t), \boldsymbol{\xi}(t)],
$$

where $\boldsymbol{x i}(t)$ is the noise associated with control input $\mathbf{u}(t)$. For simplicity, assume that $\boldsymbol{\xi}:[0, \infty) \rightarrow \mathbb{R}^{2}$ is any measurable (in the sense of Lebesgue) stochastic process taking values from the closed (Euclidean) ball $\mathcal{B}_{u}\left(\overline{\boldsymbol{\xi}}, r_{1}^{\prime}\right)$ defined by

$$
\mathcal{B}_{u}\left(\overline{\boldsymbol{\xi}}, r_{1}^{\prime}\right)=\left\{\boldsymbol{\xi}(t) \in \mathbb{R}^{2}: \| \boldsymbol{\xi}(t)-\overline{\boldsymbol{\xi}}\right\} \leq r_{1}^{\prime} \|,
$$

where $r_{1}^{\prime}>0$ is the radius of the noise associated with the robot's speed and $\overline{\boldsymbol{\xi}}$ is the mean of $\boldsymbol{\xi}(t)$, for $t \in \mathcal{I}$.

However, due to the speed limits of the wheels, the inputs are constrained as

$$
\left|u_{L}(t)\right| \leq u_{L}^{\max } \text { and }\left|u_{R}(t)\right| \leq u_{R}^{\max }, t \in \mathcal{I} .
$$

In other words, $\mathbf{u}(t)$ must be chosen from a set of admissible speeds, $\mathcal{U}_{\mathrm{ad}}$, i.e., $\quad \mathbf{u}(t) \in \mathcal{U}_{\mathrm{ad}}$. Note that a DDMR is a nonholonomic system with the nonholonomic constraint given by

$$
\dot{x} \sin \theta-\dot{y} \cos \theta=0,
$$

which ensures the wheel's non-slip movement in the lateral direction. Let us assume that the workspace has $s^{\prime}$ RF sensors and robot's signal strength measurements are modeled as the line-of-sight distance $d_{j}$ between the robot and the sensor $j, j=1, \ldots, s^{\prime}$, where $d_{j}=\sqrt{\left(x(t)-x_{t}^{j}\right)^{2}+\left(y(t)-y_{t}^{j}\right)^{2}}$. Following [26], [27], [28], we model the robot's sensory capability as the fourth-order polynomial function of $d_{j}$ defined as

$$
\rho\left(d_{j}\right)= \begin{cases}\frac{\bar{m}}{\bar{d}^{4}}\left(d_{j}^{2}-\bar{d}^{2}\right)^{2} & \text { if } d_{j} \leq \bar{d} \\ 0 & \text { if } d_{j}>\bar{d}\end{cases}
$$

where $\bar{m}$ is the robot's peak sensory capability when it is located right at the $j$ th sensor position $\left(x_{t}^{j}, y_{t}^{j}\right)$ and $\bar{d}$ is the robot's maximum sensory range from which it can receive signal strength measurements from RF sensors. Due to the robot's limited sensory range capability, it receives measurements from only a subset $s$ of the total number of RF sensors $s^{\prime}$ in the workspace, i.e., $s \leq s^{\prime}$. Since the measurements are subject to noise, the collection of measurements can be modeled as the vector of robot's sensory capabilities from $s$ sensors defined by

$$
\mathbf{z}(t)=\mathbf{h}[\mathbf{q}(t)]+\boldsymbol{\zeta}(t),
$$

where $\mathbf{z}(t) \in \mathbb{R}^{s}$ is the robot's sensory capability vector from $s$ $\mathrm{RF}$ sensors in the environment and the noise $\zeta:[0, \infty) \rightarrow \mathbb{R}^{s}$ is defined such that

$$
\mathcal{B}_{m}\left(\overline{\boldsymbol{\zeta}}, r_{2}^{\prime}\right)=\left\{\boldsymbol{\zeta}(t) \in \mathbb{R}^{s}:\|\boldsymbol{\zeta}(t)-\overline{\boldsymbol{\zeta}}\| \leq r_{2}^{\prime}\right\},
$$

where $r_{2}^{\prime}>0$ is the radius of the noise associated with the signal strength measurements and $\bar{\zeta}$ is the mean of $\boldsymbol{\zeta}(t)$, for $t \in \mathcal{I}$. The nonlinear function $\mathbf{h}[\mathbf{q}(t)] \in \mathbb{R}^{s}$ is given by

$$
\mathbf{h}[\mathbf{q}(t)]=\left[\begin{array}{lll}
\rho\left(d_{1}\right) & \ldots & \rho\left(d_{s}\right)
\end{array}\right]^{T} .
$$

Fig. 1 shows an example of the robot's sensory capability over its workspace of dimension $16 \times 16$ mwhen it receives signal strength measurements from a sensor located at $(0,0) \mathrm{m}$. In this example, the robo's peak sensory capability $\bar{m}$ is chosen as 1 and the maximum range that the robot is able to receive signals from the sensor is $\bar{d}=4 \mathrm{~m}$.

\section{Problem Statement}

Let us define the robot's control input $\mathbf{u}(t)$ as the feedback model defined by

$$
\mathbf{u}(t)=\chi[\mathbf{z}(t)]
$$

subject to (3), where $\chi[\cdot]$, is a function that takes sensory measurements as the feedback information. Substituting (7) in (1), we can formulate the robot's measurement-feedback system as

$$
\dot{\mathbf{q}}(t)=\frac{r}{2} \mathbf{B}[\mathbf{q}(t)] \chi[\mathbf{z}(t)] .
$$

Let $\mathbf{q}^{d}(t)=\left[\begin{array}{lll}x^{d}(t) & y^{d}(t) & \theta^{d}(t)\end{array}\right]^{T}$ be the desired trajectory that the robot is supposed to track and $e(t)=$ 


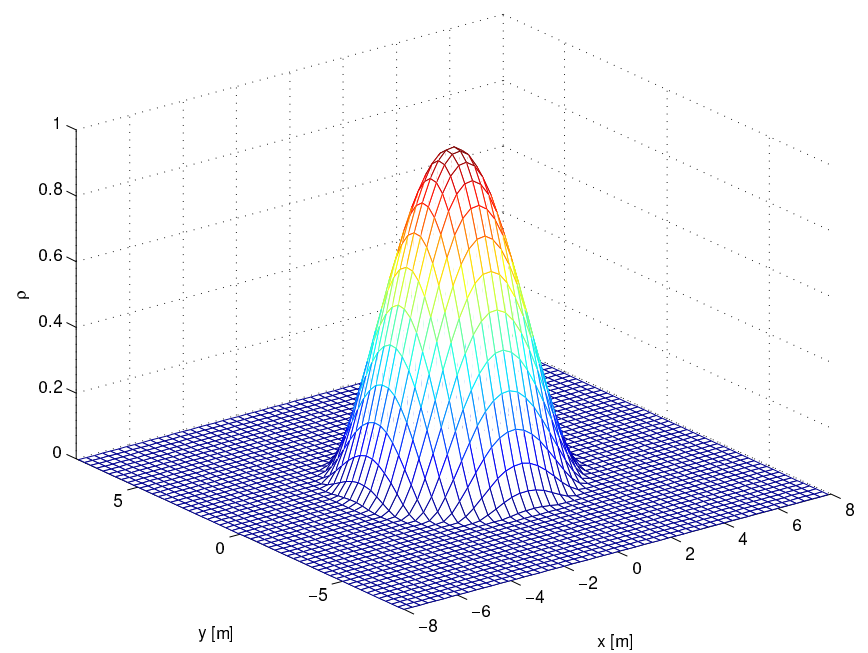

Fig. 1: Robot's sensory capability function $\rho$ with its sensory range $\bar{d}=$ 4 mand $\bar{m}=1$.

$\sqrt{\left[x^{d}(t)-x(t)\right]^{2}+\left[y^{d}(t)-y(t)\right]^{2}}$ denote its position tracking error, for $t \in \mathcal{I}$. The objective is to find the optimal control input $\mathbf{u}^{*}(t) \in \mathcal{U}_{a d}$ that generates the optimal trajectory $\mathbf{q}^{*}(t) \in \mathcal{Q}$ while minimizing the total position tracking error, $\mathscr{E}$, given by

$$
\mathscr{E}=\int_{0}^{t_{f}} e(t) d t .
$$

Given the robot's noisy kinematic model (2), its nonholonimic constraint (4), and for any $\boldsymbol{\xi}(t) \in \mathcal{B}_{u}\left(\overline{\boldsymbol{\xi}}, r_{1}^{\prime}\right), \boldsymbol{\zeta}(t) \in$ $\mathcal{B}_{m}\left(\overline{\boldsymbol{\zeta}}, r_{2}^{\prime}\right)$, the problem can be stated as follows:

$$
\left(\mathbf{q}^{*}(t), \mathbf{u}^{*}(t)\right)=\underset{\left\{\mathbf{q} \in \mathcal{Q}, \mathbf{u} \in \mathcal{U}_{a d}\right\}}{\arg \min }[\mathscr{E}], \quad \forall t \in \mathcal{I} .
$$

The nominal offline solution, $\left(\mathbf{q}^{o}(t), \mathbf{u}^{o}(t)\right)$, to the problem (10) has been given in [6] using an optimal state feedback control law for the noise-free robot model (1). Authors [6] then established an neighboring optimal control to provide an online solution, $\left(\mathbf{q}^{*}(t), \mathbf{u}^{*}(t)\right)$, to the problem (10) taking into consideration the robot's noisy model (2). The neighboring optimal control law is coupled with the robot's state estimation online using noisy signal strength measurements from all RF sensors placed in the operating range. Here we emphasize that the robot receives measurements from only a subset of sensors due to its limited sensing range to determine the online solution, $\left(\mathbf{q}^{*}(t), \mathbf{u}^{*}(t)\right)$, to the problem (10), which is the main objective of the next section.

\section{Neighboring Optimal Control LaW}

The purpose of this section is to determine an neighboring optimal control law for the robot to generate optimal trajectories $\left(\mathbf{q}^{*}(t), \mathbf{u}^{*}(t)\right)$ that follow the nominal trajectories $\left(\mathbf{q}^{o}(t), \mathbf{u}^{o}(t)\right)$ using measurements from a subset of RF sensors. Let us define the actual state and control perturbations from the nominal trajectories as

$$
\Delta \mathbf{q}(t)=\mathbf{q}(t)-\mathbf{q}^{o}(t), \Delta \mathbf{u}(t)=\mathbf{u}(t)-\mathbf{u}^{o}(t), t \in \mathcal{I} .
$$

We denote $\Delta \mathbf{u}^{o}(t)$ as the neighboring optimal control law that will generate optimal state perturbation $\Delta \mathbf{q}^{o}(t)$. The optimal trajectories $\left(\mathbf{q}^{*}(t), \mathbf{u}^{*}(t)\right)$ are then determined as follows:

$$
\mathbf{q}^{*}(t)=\mathbf{q}^{o}(t)+\Delta \mathbf{q}^{o}(t), \mathbf{u}^{*}(t)=\mathbf{u}^{o}(t)+\Delta \mathbf{u}^{o}(t), t \in \mathcal{I} .
$$

The problem now boils down to solving for the neighboring optimal control input $\Delta \mathbf{u}^{o}(t)$. For that, we define the cost functional

$$
\begin{gathered}
J(\Delta \mathbf{u})=\frac{1}{2} \Delta \mathbf{q}^{T}\left(t_{f}\right) \mathbf{P}\left(t_{f}\right) \Delta \mathbf{q}\left(t_{f}\right)+ \\
\frac{1}{2} \int_{0}^{t_{f}}\left[\Delta \mathbf{q}^{T}(t) \mathbf{Q}(t) \Delta \mathbf{q}(t)+\Delta \mathbf{u}^{T}(t) \mathbf{R}(t) \Delta \mathbf{u}(t)\right] \mathrm{d} t,
\end{gathered}
$$

where the $\mathbf{P}\left(t_{f}\right), \mathbf{Q}(t)$, and $\mathbf{R}(t)$ are positive definite matrices of appropriate dimensions. Assuming that the actual trajectories $(\mathbf{q}(t), \mathbf{u}(t))$ are the neighborhood of the nominal trajectories $\left(\mathbf{q}^{o}(t), \mathbf{u}^{o}(t)\right)$, we linearize the robot's noisy model (2) around the nominal trajectories $\left(\mathbf{q}^{o}(t), \mathbf{u}^{o}(t)\right)$ as

$\Delta \dot{\mathbf{q}}(t)=\mathbf{F}(t) \Delta \mathbf{q}(t)+\mathbf{G}(t) \Delta \mathbf{u}(t)+\mathbf{L}(t) \boldsymbol{\xi}(t), \Delta \mathbf{q}(0)=\Delta \mathbf{q}_{0}$,

where $\mathbf{F}(t)=\frac{\partial \mathbf{f}}{\partial \mathbf{q}}, \mathbf{G}(t)=\frac{\partial \mathbf{f}}{\partial \mathbf{u}}$, and $\mathbf{L}(t)=\frac{\partial \mathbf{f}}{\partial \boldsymbol{\xi}}$. The neighboring optimal trajectories $\left(\Delta \mathbf{q}^{o}(t), \Delta \mathbf{u}^{o}(t)\right)$ can then be obtained by solving

$$
\left(\Delta \mathbf{q}^{o}(t), \Delta \mathbf{u}^{o}(t)\right)=\underset{\Delta \mathbf{q}(t), \Delta \mathbf{u}(t)}{\arg \min }[J(\Delta \mathbf{u})],
$$

subject to the linear noisy perturbed model (14) and rangelimited noisy measurements from a subset of RF sensors modeled by (5). The standard solution to the problem (15) follows from the similar techniques given in [6], [29, Ch. 3], when the robot simultaneously receives measurements from all $\mathrm{RF}$ sensors placed in its operating range. This is summarized below.

Theorem 1 (Adapted from [29]): Consider the robot's linear kinematic model (14) and its quadratic cost functional given by (13). The optimal linear-quadratic state feedback control law is given by

$$
\Delta \mathbf{u}^{o}(t)=-\mathbf{R}^{-1}(t) \mathbf{G}^{T}(t) \mathbf{P}(t) \Delta \mathbf{q}(t)=-\mathbf{C}(t) \Delta \mathbf{q}(t),
$$

where $\Delta \mathbf{q}(t)=\hat{\mathbf{q}}(t)-\mathbf{q}^{o}(t)$ with $\hat{\mathbf{q}}(t)$ being the robot's estimated state taking into account its process noise and measurement noise from RF sensors. The neighboring-optimal control gain matrix $\mathbf{C}(t)$ is given by

$$
\mathbf{C}(t)=\mathbf{R}^{-1}(t) \mathbf{G}^{T}(t) \mathbf{P}(t)
$$

and $\mathbf{P}(t)$ is the solution of the differential matrix Riccati equation

$$
\begin{aligned}
\dot{\mathbf{P}}=-\mathbf{F}^{T}(t) \mathbf{P}(t)-\mathbf{Q}(t)-\mathbf{P}(t) \mathbf{F}(t)+ & \\
& \mathbf{P}(t) \mathbf{G}(t) \mathbf{R}^{-1}(t) \mathbf{G}^{T}(t) \mathbf{P}(t), \quad \mathbf{P}\left(t_{f}\right)=\mathbf{P}_{f} .
\end{aligned}
$$

It is interesting to note that the solution for $\mathbf{P}(t)$ and, therefore, for $\mathbf{C}(t)$ is independent of $\Delta \mathbf{q}(t)$. Variations in $\Delta \mathbf{q}(0)$ or $\Delta \mathbf{q}\left(t_{f}\right)$ have no effect on $\mathbf{C}(t)$, although the linear-optimal control history obviously is affected by state 
perturbations [29]. From Theorem 1, it is clear that once the solution of the differential matrix Riccati equation (18) is available, the feedback control law given by (16) can be formally constructed. The total optimal control given in (12) is then formed as the sum of the nominal and the perturbation optimal controls as:

$$
\mathbf{u}^{*}(t)=\mathbf{u}^{o}(t)+\Delta \mathbf{u}^{o}(t)=\mathbf{u}^{o}(t)-\mathbf{C}(t)\left[\hat{\mathbf{q}}(t)-\mathbf{q}^{o}(t)\right],
$$

where $\hat{\mathbf{q}}(t)$ is the robot's estimated pose. The details of determining estimated state $\hat{\mathbf{q}}(t)$ is given in [6]. Given the state estimate $\hat{\mathbf{q}}(t)$, we substitute the perturbed optimal control (16) in (14) yields the perturbed state feedback system

$$
\begin{aligned}
\Delta \dot{\mathbf{q}}^{o}(t) & =\left[\mathbf{F}(t)-\mathbf{G}(t) \mathbf{R}^{-1}(t) \mathbf{G}^{T}(t) \mathbf{P}(t)\right] \Delta \mathbf{q}^{o}(t), \\
& \equiv \mathbf{A}(t) \Delta \mathbf{q}^{o}(t), \Delta \mathbf{q}(0)=\Delta \mathbf{q}_{0} \neq \mathbf{0},
\end{aligned}
$$

with $\mathbf{A}(t) \equiv\left[\mathbf{F}(t)-\mathbf{G}(t) \mathbf{R}^{-1}(t) \mathbf{G}^{T}(t) \mathbf{P}(t)\right]$ and the corresponding neighboring state trajectory can then be described by

$$
\Delta \mathbf{q}(t)=\boldsymbol{\Phi}(t) \Delta \mathbf{q}_{0},
$$

where $\boldsymbol{\Phi}(t)=e^{t A(t)}$ is the state transition matrix. The feedback model (20) with the quadratic cost functional (13) is similar to the optimal linear quadratic regulator problem, which is stable in the Lyapunov sense [30]. In other words, the optimality leads to stability.

The following steps summarize computing the robot's optimal trajectory $\left(\mathbf{q}^{*}(t), \mathbf{u}^{*}(t)\right)$ :

1) Define the desired trajectory $\mathbf{q}^{d}(t), t \in \mathcal{I}$, that the robot is supposed to follow.

2) Compute the robot's nominal optimal trajectory $\left(\mathbf{q}^{o}(t), \mathbf{u}^{o}(t)\right), \quad t \in \mathcal{I}$, using the matrix-valued statefeedback control law as illustrated in [6],

3) Solve the matrix-Riccati equation (18) for $\mathbf{P}(t)$ backward in time.

4) Employ sensory measurements using the range-limited sensor model (5) to estimate the robot's pose $\hat{\mathbf{q}}(t), t \in$ $\mathcal{I}$, by following the optimal filter given in [6].

5) Compute the neighboring-optimal control gain matrix $\mathbf{C}(t)$ from (17) subject to the robot's linear kinematic constraint (14) around the estimated states $\hat{\mathbf{q}}(t)$.

6) Determine the neighboring optimal control $\Delta \mathbf{u}^{o}(t)$ using (16) and the corresponding neighboring optimal state $\Delta \mathbf{q}^{o}(t)$ from (20).

7) Use (19) to compute the total optimal control input $\mathbf{u}^{*}(t)$.

8) Apply $\mathbf{u}^{*}(t)$ in the robot model (1) to determine the optimal trajectory $\mathbf{q}^{*}(t)$ for every $t \in \mathcal{I}$.

Numerical results implementing the above steps are given in the following section.

\section{Simulation Results}

In this section, we illustrate the robot's tracking performance given the fact that the robot has rangelimited sensory capabilities. For that, a virtual circular-shaped mobile robot with the wheel base of $l=30 \mathrm{~cm}$ and the radius of each wheel $r=8.25 \mathrm{~cm}$ is employed. The robot's wheel speeds are constrained as $u_{R}^{\max }=u_{L}^{\max }=10 \mathrm{rad} \cdot \mathrm{s}^{-1}$. The performance metrics adopted in this work are the robot's pose (position and orientation) tracking error given by

$$
\mathbf{q}^{*}(t)-\mathbf{q}^{o}(t)=\mathbf{q}_{e}(t)=\left[\begin{array}{l}
x_{e}(t) \\
y_{e}(t) \\
\theta_{e}(t)
\end{array}\right]=\left[\begin{array}{l}
x^{*}(t)-x^{o}(t) \\
y^{*}(t)-y^{o}(t) \\
\theta^{*}(t)-\theta^{o}(t)
\end{array}\right]
$$

and the average cumulative position error defined in (9) over the time interval of $\mathcal{I} \equiv[0,60] s$, which allow us to make quantitative assessment of the proposed neighboring optimal controller. The dimension of the virtual test area is about $16 \times 16 \times 3 \mathrm{~m}$, where $25 \mathrm{RF}$ sensors $\left(s^{\prime}=25\right)$ are uniformly placed on the floor of the workspace (denoted by $x$ 's in the figures below). The nominal trajectory $\mathbf{q}^{o}(t)$ and control $\mathbf{u}^{o}(t)$ are computed offline according the state-feedback control law illustrated in [6]. The sampling time period for the simulation is set to $0.1 \mathrm{~s}$. The mean and standard deviation of the robot's actuator noise, $\boldsymbol{\xi}(t)$, are chosen to be 0 and $0.1 \mathrm{rad} \cdot \mathrm{s}^{-1}$, respectively. As for the noise $\boldsymbol{\zeta}(t)$ of sensory measurements modeled by (5), these quantities are 0 and 0.2 . For the robot's sensory model (5), we use $\bar{m}=1$ and $\bar{d}=4 \mathrm{~m}$.

The robot's initial estimated position is at $(-7,-3) \mathrm{m}$, with an orientation $5.7^{\circ}$ whereas its nominal position is at $(-6,-2) \mathrm{m}$ with an orientation $0^{\circ}$. The performance of the neighboring optimal controller in tracking the robot's nominal trajectory $\mathbf{q}^{o}(t)$ is summarized in Fig. 2 which is the straightforward implementation of the neighboring optimal control law (19). Note that the robot's pose is estimated using the optimal filter developed in [6] except that the range-limited sensor model is applied in the current work. The robot's actual and nominal pose (position and orientation) is given Fig. 2(a), where the dashed and solid lines are, respectively, the robot's actual and nominal trajectories with the corresponding pose error shown in Fig. 2(c). The initial error in Fig. 2(c) is due to the robot's initial state estimation error since it receives measurements from about four sensors (see Fig. 2(b)). The robot's sensory range is represented by the circle of radius $\bar{d}=4 \mathrm{~m}$ as shown in Figs. 2(b)-2(f). At about $t=30 \mathrm{~s}$, the robot receives signals from about seven sensors as shown in Fig. 2(d). As expected, the estimation error is practically nil about this time (see Fig. 2(c)). Note that as the robot moves to track the nominal trajectory, it gradually receives measurements from less number of RF sensors as it is clear from Fig. 2(f). It is quite interesting to notice that robot is still able to track the nominal trajectory with tracking error of practically nil (see Fig. 2(c)). Fig. 2(e) reveals the time history of the robots left and right wheel speeds. It is clear that the speeds are within the maximum limit $\pm 10 \mathrm{rad} \cdot \mathrm{s}^{-1}$ as expected.

\section{CONCLUSION}

In this paper, we presented a neighboring optimal control law for a mobile robot to track a pre-defined trajectory with its range-limited sensory capabilities. The robot receives signal strength measurement of RF sensors placed on 2-D 

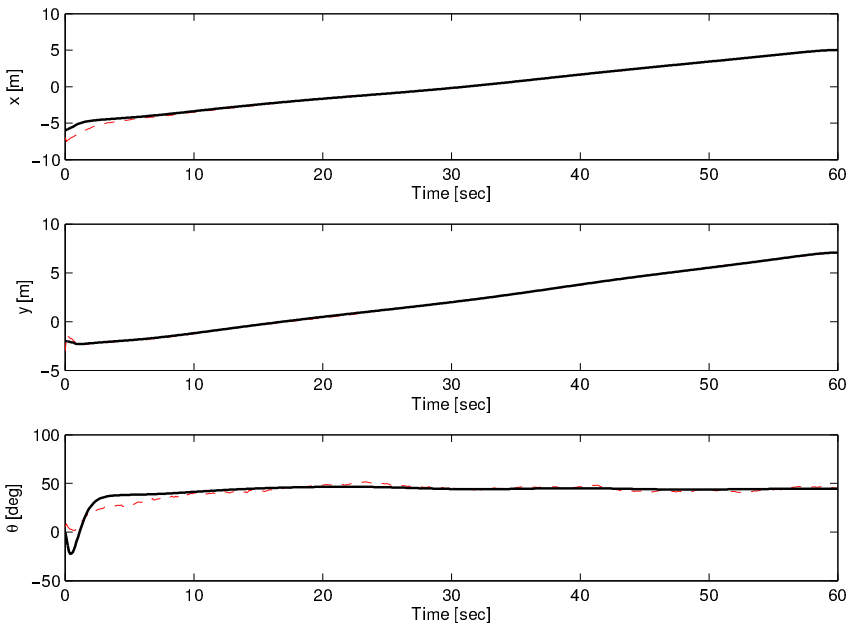

(a)

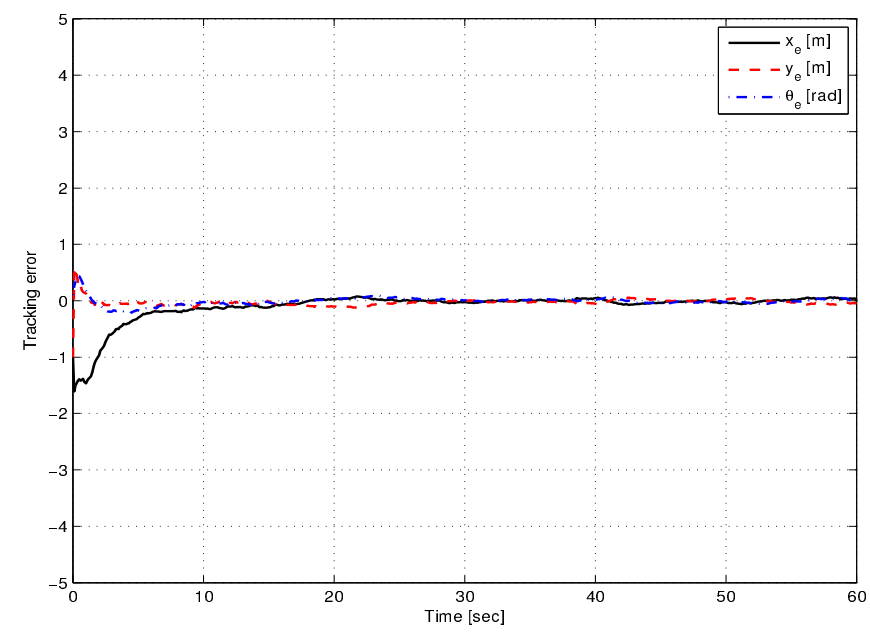

(c)

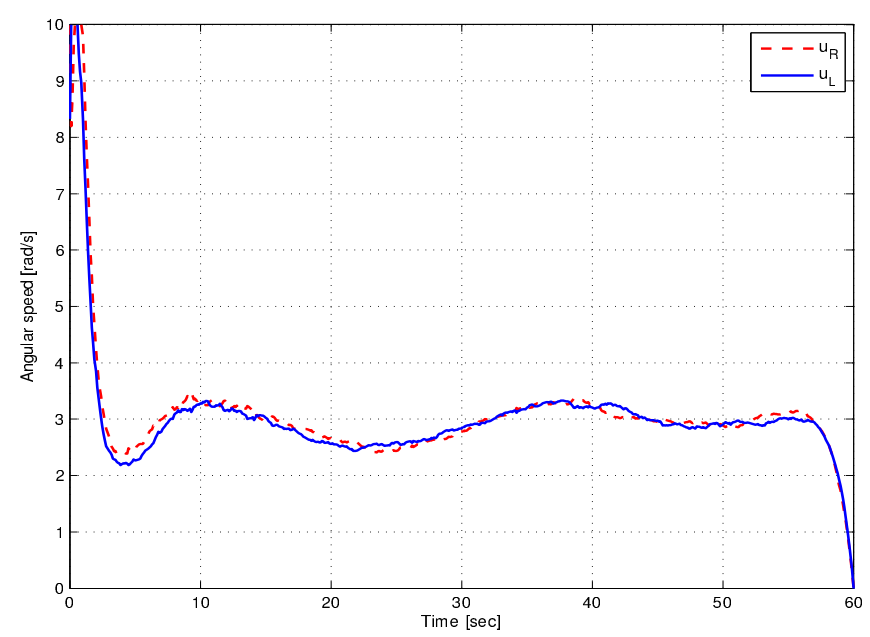

(e)

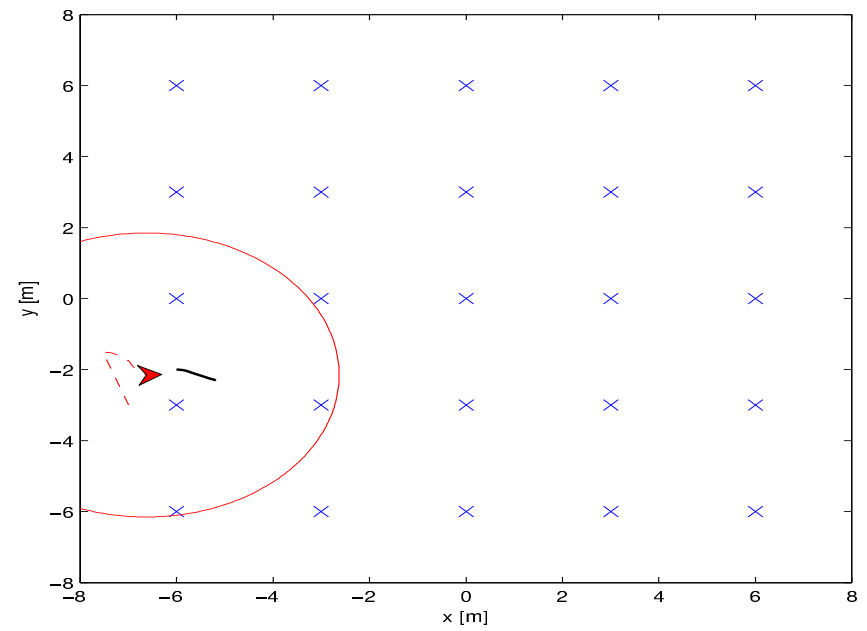

(b)

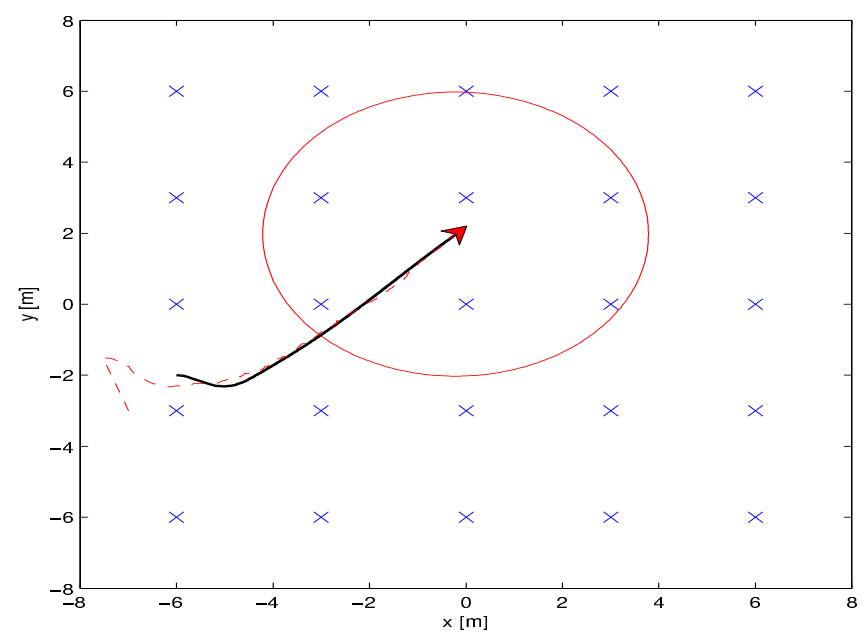

(d)

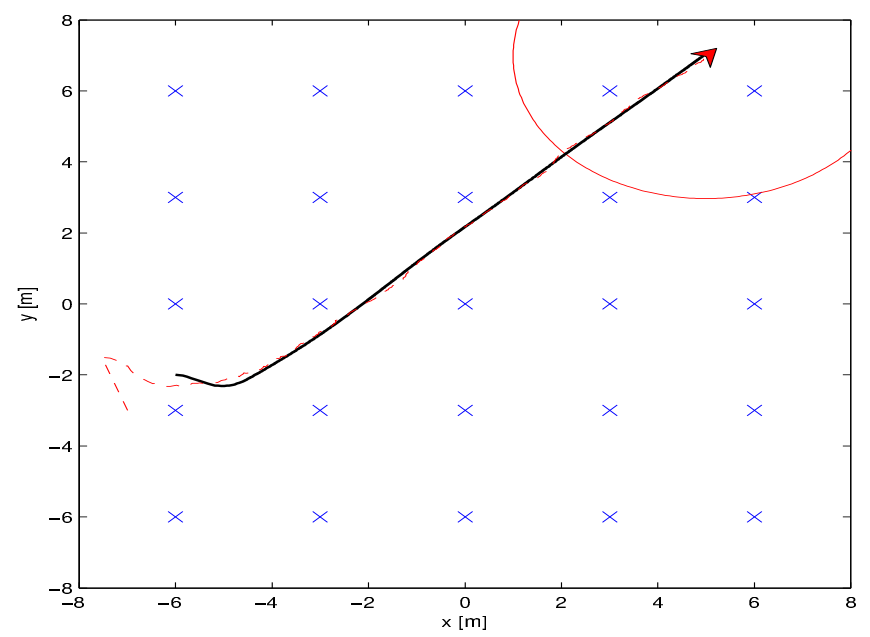

(f)

Fig. 2: Controller's performance in tracking the robot's nominal trajectory, (a) robot's pose, (c) robot's tracking error, and (e) control inputs (wheel speeds); and the robot's trajectories at time (b) $t=0.2 \mathrm{~s}$, (d) $t=30 \mathrm{~s}$, and (f) $t=59 \mathrm{~s}$. 
environment and estimates its states based on the noise sensory model. The robots states are partially observed simply because it estimates its position and orientation based on signal strength measurements coming from RF sensors. The proposed controller is the direct consequence of our previously published article but this paper shows how a mobile robot tracks a predefined trajectory when RF sensors are placed on 2-D positions and robot receives only signal strength measurements from a subset of RF sensors due its range-limited capabilities.

\section{REFERENCES}

[1] D. Sun, C. Wang, W. Shang, and G. Feng, "A synchronization approach to trajectory tracking of multiple mobile robots while maintaining timevarying formations," IEEE Transactions on Robotics, vol. 25, no. 5, pp. 1074-1086, Oct. 2009.

[2] R. Cui, S. S. Ge, B. V. E. How, and Y. S. Choo, "Leader-follower formation control of underactuated autonomous underwater vehicles," Ocean Engineering37(2010)14911502, vol. 37, no. 1, pp. 1491-1502, Sep. 2010

[3] A. Y. Alanis, M. Lopez-Franco, N. Arana-Daniel, and C. Lopez-Franco, "Discrete-time neural control for electrically driven nonholonomic mobile robots," Journal of Adaptive Control and Signal Processing, vol. 26, no. 7, pp. 630-44, Jul. 2012.

[4] Z. Li, C. Yang, and Y. Tang, "Decentralised adaptive fuzzy control of coordinated multiple mobile manipulators interacting with non-rigid environments," IET Control Theory and Applications, vol. 7, no. 3, pp. 397-410, Feb. 2013.

[5] C. Yang, Z. Li, and J. Li, "Trajectory planning and optimized adaptive control for a class of wheeled inverted pendulum vehicle models," IEEE Transactions on Cybernetics, vol. 43, no. 1, pp. 24-36, Jan. 2013.

[6] M. Miah and W. Gueaieb, "RFID-based mobile robot trajectory tracking and point stabilization through on-line neighboring optimal control," Journal of Intelligent \& Robotic Systems, pp. 1-23, 2014. [Online]. Available: http://dx.doi.org/10.1007/s10846-014-0048-3

[7] D. Nganga-Kouya and F. Okou, "Adaptive backstepping control of a wheeled mobile robot," in 17th Mediterranean Conference on Control and Automation, Piscataway, NJ, USA, 2009, pp. 85-91.

[8] D. Chwa, "Tracking control of differential-drive wheeled mobile robots using a backstepping-like feedback linearization," IEEE Transactions on Systems, Man and Cybernetics, Part A: Systems and Humans, vol. 40, no. 6, pp. 1285-1295, Nov. 2010.

[9] Q. Cui, X. Li, X. Wang, and M. Zhang, "Backstepping control design on the dynamics of the omni-directional mobile robot," Applied Mechanics and Materials, vol. 203, pp. 51-6, 2012.

[10] D. Chwa, "Sliding-mode tracking control of nonholonomic wheeled mobile robots in polar coordinates," IEEE Transactions on Control Systems Technology, vol. 12, no. 4, pp. 637-644, Jul. 2004.

[11] C.-L. Hwang and N.-W. Chang, "Fuzzy decentralized sliding-mode control of a car-like mobile robot in distributed sensor-network spaces," IEEE Transactions on Fuzzy Systems, vol. 16, no. 1, pp. 97-109, 2008.

[12] M. Rubagotti, M. Della Vedova, and A. Ferrara, "Time-optimal slidingmode control of a mobile robot in a dynamic environment," IET Control Theory \& Applications, vol. 5, no. 16, pp. 1916-24, 2011.

[13] H. Chen, M.-M. Ma, H. Wang, Z.-Y. Liu, and Z.-X. Cai, "Moving horizon $\mathcal{H}_{\infty}$ tracking control of wheeled mobile robots with actuator saturation," IEEE Transactions on Control Systems Technology, vol. 17, no. 2, pp. 449-57, Mar. 2009.
[14] B. Kim, D. Necsulescu, and J. Sastadek, "Autonomous mobile robot model predictive control," International Journal of Control, vol. 77, no. 16, pp. 1438-45, 2004.

[15] R. Gonzalez, M. Fiacchini, T. Alamo, J. Guzman, and F. Rodriguez, "Online robust tube-based mpc for time-varying systems: a practical approach," International Journal of Control, vol. 84, no. 6, pp. 115770, 2011.

[16] P. Morin and C. Samson, "Control of nonholonomic mobile robots based on the transverse function approach," IEEE Transactions on Robotics, vol. 25, no. 5, pp. 1058-73, Oct. 2009.

[17] M. Michalek and K. Kozowski, "Vector-field-orientation feedback control method for a differentially driven vehicle," IEEE Transactions on Control Systems Technology, vol. 18, no. 1, pp. 45-65, jan 2010.

[18] S. Park and S. Hashimoto, "Autonomous mobile robot navigation using passive RFID in indoor environment," IEEE Transactions on Industrial Electronics, vol. 56, no. 7, pp. 2366-2373, 2009.

[19] B.-S. Choi, J.-W. Lee, J.-J. Lee, and K.-T. Park, "A hierarchical algorithm for indoor mobile robot localization using RFID sensor fusion," IEEE Transactions on Industrial Electronics, vol. 58, no. 6, pp. 22262235,2011

[20] S. Han, H. Lim, and J. Lee, "An efficient localization scheme for a differential-driving mobile robot based on rfid system," IEEE Transactions on Industrial Electronics, vol. 54, no. 6, pp. 3362-3369, dec 2007.

[21] R. Luo, C.-T. Chuang, and S.-S. Huang, "RFID-based indoor antenna localization system using passive tag and variable RF-attenuation," in IEEE International Conference on Industrial Electronics Society, nov 2007, pp. 2254-2259.

[22] L. Jing and P. Yang, "A localization algorithm for mobile robots in rfid system," in International Conference on Wireless Communications, Networking and Mobile Computing, sept. 2007, pp. 2109-2112.

[23] K. Kodaka, H. Niwa, Y. Sakamoto, M. Otake, Y. Kanemori, and S. Sugano, "Pose estimation of a mobile robot on a lattice of rfid tags," in IEEE/RSJ International Conference on Intelligent Robots and Systems, sept. 2008, pp. 1385-1390.

[24] J. H. Lee, C. Lin, H. Lim, and J. M. Lee, "Sliding mode control for trajectory tracking of mobile robot in the RFID sensor space," International Journal of Control, Automation and System, vol. 7, no. 3, pp. 429-435, Jun. 2009.

[25] W. Gueaieb and M. S. Miah, "An intelligent mobile robot navigation technique using RFID technology," IEEE Transactions on Instrumentation and Measurement, vol. 57, no. 9, pp. 1908-1917, September 2008.

[26] I. I. Hussein and D. Stipanovic, "Effective coverage control using dynamic sensor networks," in IEEE Conference on Decision and Control, dec 2006, pp. 2747-2752.

[27] — , "Effective coverage control for mobile sensor networks with guaranteed collision avoidance," IEEE Transactions on Control Systems Technology, Special Issue Multi-Vehicle System, Cooperative Control Applications, vol. 15, no. 4, pp. 642-657, 2007.

[28] Y. Wang and I. I. Hussein, "Awareness coverage control over largescale domains with intermittent communications," IEEE Transactions on Automatic Control, vol. 55, no. 8, pp. 1850-1859, 2010.

[29] R. F. Stengel, Optimal Control and Estimation. Dover publications, inc., 1994.

[30] N. U. Ahmed, Dynamic Systems and Control with Applications. New Jersey: World Scientific, 2006. 